\title{
An Intraprostatic Modified Release Formulation of Antiandrogen 2-Hydroxyflutamide for Localized Prostate Cancer
}

\section{Tammela, Teuvo L.}

2017-12

Tammela , T L , Häggman , M , Ladjevardi , S , Taari , K, Isotalo , T , Lennernäs , H , Weis , J , von Below , C , Wassberg , C , Lennernäs , B , Tolf , A, Axén , N, Gölander , C-G \& Ahlström , H 2017 , ' An Intraprostatic Modified Release Formulation of Antiandrogen 2-Hydroxyflutamide for Localized Prostate Cancer ' , Journal of Urology , vol. 198 , no. 6 , pp. 1333-1339 . https://doi.org/10.1016/j.juro.2017.07.072

http://hdl.handle.net/10138/298170

https://doi.org/10.1016/j.juro.2017.07.072

publishedVersion

Downloaded from Helda, University of Helsinki institutional repository.

This is an electronic reprint of the original article.

This reprint may differ from the original in pagination and typographic detail.

Please cite the original version. 


\title{
An Intraprostatic Modified Release Formulation of Antiandrogen 2-Hydroxyflutamide for Localized Prostate Cancer
}

\author{
Teuvo L. Tammela, Michael Häggman, Sam Ladjevardi, Kimmo Taari, Taina Isotalo, \\ Hans Lennernäs, Jan Weis, Catrin von Below, Cecilia Wassberg, Bo Lennernäs, \\ Anna Tolf, Niklas Axén, ${ }^{*}$ Carl-Gustaf Gölander and Håkan Ahlström†
}

\begin{abstract}
From the Departments of Urology, Tampere University Hospital and University of Tampere (TLT), Tampere, Helsinki University Hospital (KT), Helsinki and Päijät-Häme Central Hospital (TI), Lahti, Finland, and Department of Urology, Uppsala University Hospital (MH, SL), Radiology, Department of Surgical Sciences (JW, CVB, CW) and Departments of Pharmacy (HL) and Immunology Genetics and Pathology (AT), Uppsala University and LIDDS AB (NA, CGG), Uppsala and Department of Oncology, University of Örebro (BL), Örebro, Sweden
\end{abstract}

Purpose: We investigated the tolerability, safety and antitumor effects of a novel intraprostatic depot formulation of antiandrogen 2-hydroxyflutamide (in NanoZolid $\left.{ }^{\circledR}\right)$ in men with localized prostate cancer.

Materials and Methods: Two clinical trials, LPC-002 and LPC-003, were performed in a total of 47 men. The formulation was injected transrectally into the prostate under ultrasound guidance. In LPC-002 the effects on prostate specific antigen and prostate volume were measured for 6 months in 24 patients. In LPC003 antitumor effects were evaluated by histopathology and magnetic resonance imaging including spectroscopy during 6 or 8 weeks in 23 patients. In each study testosterone and 2-hydroxyflutamide in plasma were measured as well as quality of life parameters.

Results: In LPC-002 (mean dose $690 \mathrm{mg}$ ) a reduction was observed in prostate specific antigen and prostate volume. Average nadir prostate specific antigen and prostate volume were $24.9 \%$ and $14.0 \%$ below baseline, respectively. When increasing the dose in LPC-003 to 920 and 1,740 mg, average prostate specific antigen decreased $16 \%$ and $23 \%$ after 6 and 8 weeks, respectively. Magnetic resonance imaging and magnetic resonance spectroscopy showed morphological changes and a global reduction in metabolite concentrations following treatment, indicating an antitumor response. Injections did not result in hormone related side effects. Three serious adverse events were reported and all resolved with oral antibiotic treatment.

Conclusions: Intraprostatic injections of 2-hydroxyflutamide depot formulations showed antitumor effects, and proved to be safe and tolerable. However, for better anticancer effects higher doses and better dose distribution are suggested.

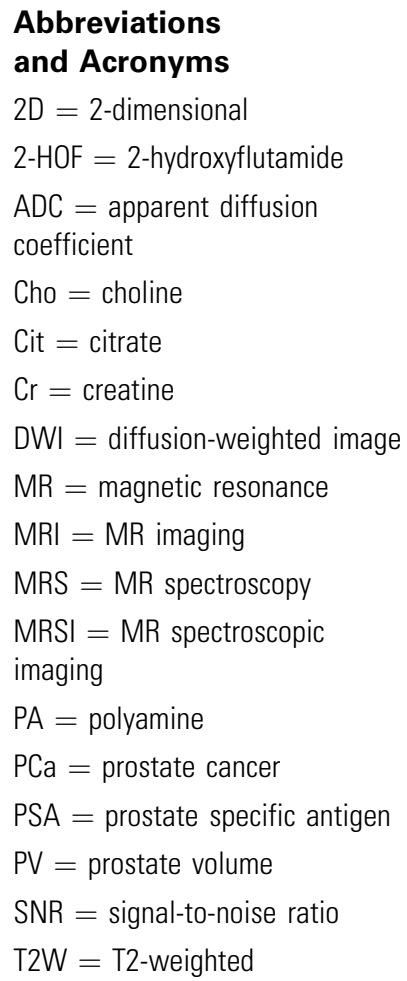
review board, ethics committee or ethical review board study approval; principles of Helsinki Declaration were followed in lieu of formal ethics committee approval; institutional animal care and use committee approval; all human subjects provided written informed consent with guarantees of confidentiality; IRB approved protocol number; animal approved project number.

* Financial interest and/or other relationship with LIDDS AB.

† Correspondence: Department of Surgical Sciences, Radiology, Uppsala University Hospital, SE-75185, Uppsala, Sweden (telephone: +4618-6114771; e-mail: hakan.ahlstrom@radiol.uu.se). 
Key Words: prostatic neoplasms, prostate-specific antigen, androgen antagonists, delayed-action preparations, calcium sulfate

Prostate cancer is the second most common cancer and the fifth leading cause of cancer death in men worldwide. In 2015 approximately 1.5 million men were diagnosed with $\mathrm{PCa}$ worldwide, of whom most were older than 65 years. Around $90 \%$ of them had localized (stage I or II) prostate cancer. ${ }^{1-3}$ Improved diagnostic techniques, including PSA, result in earlier diagnosis of $\mathrm{PCa}$ and earlier treatment. Consequently the treatment regimen for $\mathrm{PCa}$ has changed and curative treatment with prostatectomy and radiation has become more frequent. ${ }^{4,5}$ However, curative and systemic palliative treatments are often associated with negative side effects such as incontinence, rectal toxicity, loss of libido and gynecomastia. $^{6-8}$

A significant PCa group is elderly patients with localized low or medium grade disease who often have significant comorbidities and are on systemic antiandrogen therapy with troublesome hormonal side effects. ${ }^{5,9}$ These men would benefit from a less invasive local antiandrogen treatment. Such local treatment would potentially also be beneficial when used as neoadjuvant prior to radiotherapy to reduce prostate volume and thereby increase surgical precision. Today when most patients diagnosed with $\mathrm{PCa}$ are assigned to active surveillance, there is a risk of under treatment. Local antiandrogen treatment may offer a means to halt further progression.

Accordingly if proven effective, there is a need for local antiandrogen treatment to halt PCa progression without resulting in the negative side effects of systemic androgen therapies. ${ }^{10}$ For such purposes local pharmaceutical injections in the prostate have already been described. ${ }^{11-13}$ Liproca ${ }^{\circledR}$ Depot is a modified release formulation under development. The product is composed of $2-\mathrm{HOF}$, the active metabolite of the antiandrogen flutamide, encapsulated in a calcium sulfate drug carrier matrix, NanoZolid ${ }^{\circledR}{ }^{14,15}$ Following intraprostatic injection the matrix dissolves and 2 -HOF is gradually released, providing a local drug concentration in the prostate tissue for a prolonged period.

In the current study we evaluated the tolerability, safety and antitumor effects of the investigational product after a single dose in men with localized prostate cancer.

\section{MATERIALS AND METHODS}

\section{Drug Product}

The investigational modified release product Liproca Depot is based on the NanoZolid microstructurally optimized calcium sulfate matrix as a drug carrier system for the active drug 2-HOF. The product consists of a powder with 25 weight per percent of 2-HOF in calcium sulfate and a 0.25 weight per percent of sodium carboxymethyl cellulose aqueous solution. Immediately before administration the components were mixed to a viscous suspension, which was injected transrectally under ultrasound guidance through a 17 gauge $(1.4 \mathrm{~mm})$ needle. The formulation produces an initial boost dose followed by a slower release during about 6 months. ${ }^{15}$

\section{Study Designs}

The presented results are based on data from 2 phase I/II clinical trials, LPC-002 (EudraCT [European Union Clinical Trials Register] No. 2009-010079-25) and LPC003 (EudraCT No. 2011-001137-16). Table 1 lists the main experimental parameters. Prior to these studies the uneventful low dose, first in man LPC-001 study was performed.

LPC-002 was an open, nonrandomized study performed at 3 urological centers in Finland, including Tampere and Helsinki University Hospitals, and Lahti Central Hospital. The study investigated the effects and safety of a single injection of the study drug in the most affected prostate lobe in patients with localized PCa. The nadir values of PSA and PV, defined as the lowest levels after treatment, were investigated. Patients who showed progression, defined as a $25 \%$ or greater PSA increase over baseline or nadir, within 24 weeks after the first injection (part 1) were offered a second equal dose (part 2).

Table 1. Essential LPC-002 and LPC-003 study parameters

\begin{tabular}{|c|c|c|}
\hline & LPC-002 & LPC-003 \\
\hline \multicolumn{3}{|l|}{ No. pts: } \\
\hline Part 1 & 24 & - \\
\hline Part 2 & 9 & - \\
\hline Group 1 & - & 18 \\
\hline Group 2 & - & 5 \\
\hline \multicolumn{3}{|l|}{ Study inclusion criteria: } \\
\hline Age & 45 or Greater & 45 or Greater (range $50-75$ ) \\
\hline PSA (ng/ml) & Less than 20 & Less than 20 \\
\hline Tumor stage & $\mathrm{T} 1-\mathrm{T} 2$ & $\mathrm{~T} 1 \mathrm{c}$ or $\mathrm{T} 2 \mathrm{a}-\mathrm{T} 2 \mathrm{c}$ \\
\hline Gleason score & $3+4$ or Less & $3+4$ or Less \\
\hline Main parameters & $\begin{array}{l}\text { PSA, prostate vol, } \\
\text { testosterone }\end{array}$ & $\begin{array}{c}\text { PSA, prostate vol, } \\
\text { histology, MR }\end{array}$ \\
\hline \multicolumn{3}{|l|}{ Injected vol (ml): } \\
\hline Part 1 & $2-8$ & - \\
\hline Part 2 & $3-4$ & - \\
\hline Group 1 & - & $3-10$ \\
\hline Group 2 & - & $9-12$ \\
\hline \multicolumn{3}{|l|}{$\begin{array}{l}\text { Mean mg 2-HOF } \\
\text { dose (range): }\end{array}$} \\
\hline Part 1 & $716(400-1,560)$ & - \\
\hline Part 2 & $664(600-800)$ & - \\
\hline Group 1 & - & $920 \quad(600-1,300)$ \\
\hline Group 2 & - & $1740(1,140-2,400)$ \\
\hline Injection & 1 Lobe, lesion & 1 or 2 Lobes, lesion \\
\hline Study duration (wks): & 24 & \\
\hline Group 1 & & 6 \\
\hline Group 2 & & 8 \\
\hline
\end{tabular}


Nine patients received the second injection and were monitored for another 24 weeks. Blood samples were drawn before injection (baseline), at days 1, 4 and 6, and monthly thereafter. PSA and testosterone were measured by chemiluminescence assay. For pharmacokinetic analysis of 2-HOF plasma was collected at 2, 4, 6 and 24 hours, and analyzed by liquid chromatography-tandem mass spectrometry as previously described. ${ }^{15}$ Transrectal ultrasound PV determination, I-PSS (International Prostate Symptom Score) and adverse events were assessed monthly. Quality of life was assessed by the EORTC (European Organisation for Research and Treatment of Cancer) PR25 questionnaire at weeks 4, 12 and $24 .^{16}$

LPC-003 was an open, nonrandomized, single dose study at 2 urological centers, including Tampere University Hospital in Finland and Uppsala University Hospital in Sweden. The study focused on patients with localized PCa who were scheduled for prostatectomy 6 or 8 weeks after a single or double-sided injection in major lesions. The study was performed in a first patient group (group 1) and in newly enrolled patients (group 2) who received an increased dose (table 1). The second group was added to confirm that higher doses could be administered. Target dose density was 30 and $60 \mathrm{mg} / \mathrm{ml}$ per PV in groups 1 and 2 , respectively. Blood samples were drawn before injection and at weeks 1,4 and 6 in group 1 or week 8 in group 2 to determine 2-HOF, testosterone and PSA, for which the same methods were used as in LPC-002. Prostate volume was measured before injection and at weeks 4 and 6 or 8 . Adverse events and I-PSS were assessed at each visit.

In LPC-003 standard histology was performed on biopsy specimens before drug injection. Prostatectomy specimens were fixed in $10 \%$ formalin, embedded in paraffin and sectioned from apex to base into $4 \mathrm{~mm}$ thick slices perpendicular to the rectal wall. The primary end points were histopathological changes, ie altered histological structure, an increased cytoplasm-to-nuclear ratio, nuclear hyperchromatism, visible nucleoli and clear cytoplasm.

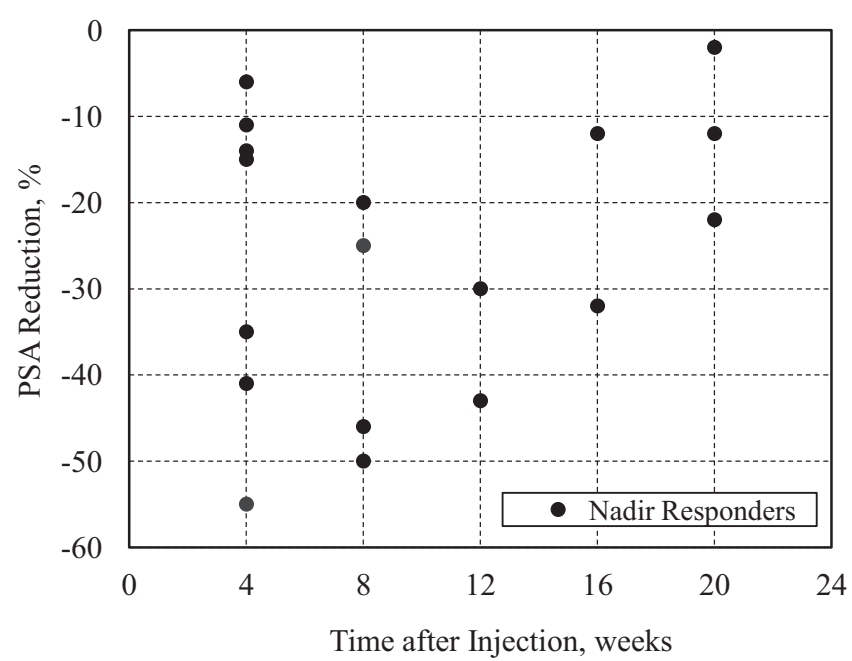

Figure 1. PSA reduction at nadir relative to screening and baseline averages in all individual responders in LPC-002.
Table 2. PSA and ultrasound prostate volume decrease relative to mean screening and baseline values

\begin{tabular}{lccc}
\hline & \multicolumn{3}{c}{ Mean \pm SD \% Decrease } \\
\cline { 3 - 4 } Study (wk) & No. Pts & PSA & Prostate Vol \\
\hline LPC-002 part 1: & & $0.00 \pm 0.00$ & $0.0 \pm 0.0$ \\
0 & 24 & $1.00 \pm 52.85$ & $-3.9 \pm 7.67$ \\
4 & 24 & $-2.18 \pm 23.88$ & $-6.3 \pm 8.35$ \\
8 & 23 & $-4.87 \pm 35.30$ & $-9.9 \pm 9.50$ \\
12 & 19 & $-6.67 \pm 16.72$ & $-15.2 \pm 7.68$ \\
16 & 17 & $-9.52 \pm 22.17$ & $-13.7 \pm 8.13$ \\
20 & 15 & $-4.53 \pm 17.20$ & $-7.3 \pm 10.7$ \\
24 & 15 & $0.00 \pm 0.00$ & $0.00 \pm 0.0$ \\
LPC-003 group 1: & & $-20.17 \pm 29.48$ & $-2.3 \pm 16.22$ \\
0 & 18 & $-15.67 \pm 29.72$ & $-13.1 \pm 12.41$ \\
4 & 17 & 0.00 & 0.00 \\
6 & 18 & 2.46 & 11.09 \\
LPC-003 group 2: & & -23.31 & -1.41 \\
0 & 5 & 5 & \\
4 & 4 & & \\
8 & & &
\end{tabular}

MRI, single voxel ${ }^{1} \mathrm{H}-\mathrm{MRS}$ and 2D MRSI of the prostate were performed as part of LPC-003, before injection and at week 6 in group 1 or at week 8 in group 2 before prostatectomy. Measurements were made with a 3 Tesla Achieva clinical scanner (Philips Medical Systems, Best, The Netherlands) using a whole body coil as excitation and a receiver phase array coil. MRI examinations included axial T1-weighted, axial, coronal and sagittal T2-weighted DWI sequences. ADCs were calculated using $5 \mathrm{~b}$-values, including $0,100,200,400$ and $500 \mathrm{~s} / \mathrm{mm}^{2}$. Also, 1 extra DWI acquisition with a single b-value of $1,000 \mathrm{~s} / \mathrm{mm}^{2}$ was used for imaging purposes. The spectroscopic methods were described previously. ${ }^{17}$

MRI was performed by 2 radiologists experienced with oncologic imaging. The functional parameters indicative of malignancy, high signal and low signal compared to the signal in apparently normal prostate tissue on DWI and $\mathrm{ADC}$ maps, respectively, were analyzed visually to detect tumor. Changes in dominant tumor signal intensity and size were assessed on T2W images before and after therapy. The presence of post-biopsy hemorrhage was

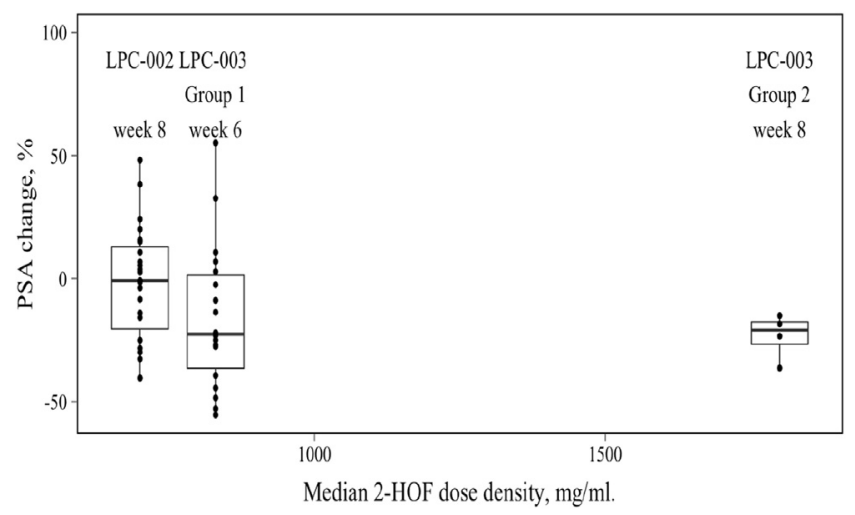

Figure 2. Median PSA decrease relative to baseline and screening averages in LPC-002 and LPC-003 for increased dose density in prostate at weeks 6 and 8 . 


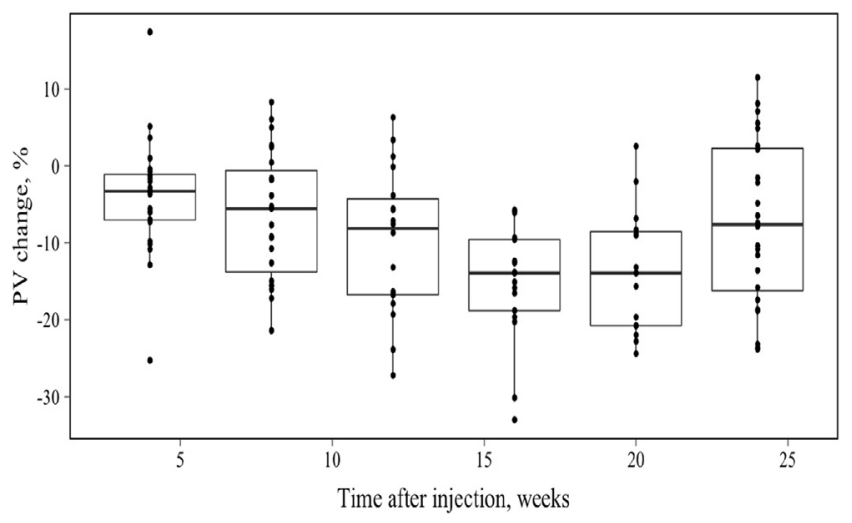

Figure 3. Median PV decrease relative to baseline and screening averages in LPC-002 part 1 vs time after injection.

assessed on T1-weighted images. MRS was assessed by quantifying the $(\mathrm{Cho}+\mathrm{PA}+\mathrm{Cr}) / \mathrm{Cit}$ spectral intensity ratio and by the SNR of each individual metabolite.

The presented studies were approved by the ethics committee at each hospital and by the national MPA (Medical Products Agency) in Sweden and Finland. All patients provided signed informed consent prior to treatment.

\section{RESULTS}

In LPC-002 a PSA nadir was noted in 20 of 24 patients (83\%) compared to mean PSA measured at screening and baseline. This subgroup was classified as responders and PSA reductions up to about $50 \%$ were reached (fig. 1). The mean \pm SD PSA nadir in all responders was $-24.9 \% \pm 13.8 \%$. Time to nadir varied between weeks 4 and 20 (mean 56 days). Table 2 lists PSA reductions in all patients in LPC-002 and LPC-003. Some nonresponders in LPC-002 showed an extraordinary increase in PSA during early followup, presumably due to tissue damage from the injection. In these patients mean
Table 3. Antiandrogen effects on histopathology, MRI and MRS in LPC-003

\begin{tabular}{lcc}
\hline & \multicolumn{2}{c}{ No. Pts/Total No. } \\
\cline { 2 - 3 } \multicolumn{1}{c}{ Observation } & Group 1 & Group 2 \\
\hline Histology & & \\
Increased cytoplasm/nuclear ratio & $10 / 18$ & $0 / 4$ \\
Cytoplasm clearing & $10 / 18$ & $0 / 5$ \\
Stroma reduction, gland clustering & $11 / 18$ & $1 / 5$ \\
Overall histopathology changes & $12 / 18$ & $1 / 5$ \\
MR effect changes: & $11 / 15$ & $3 / 4$ \\
$\quad$ Global & $6 / 15$ & $2 / 4$ \\
Tumor & $11 / 12$ & $3 / 4$ \\
1-Voxel MRS: & $5 / 5$ & - \\
Increased (Cho + PA + Cr)/Cit & $5 / 5$ & \\
$\quad$ Decreased signal/noise ratio & $5 / 5$ & \\
Overall 2D MRSI changes & & \\
\hline
\end{tabular}

PSA decreased only moderately to a minimum of $-9.5 \%$ at 5 months.

Overall in LPC-002 a PSA reduction was clearly detectable at weeks 8 to 20. In LPC-003 mean PSA was decreased $16 \%$ at week 6 in group 1 and $23 \%$ at week 8 in group 2 . However, interpatient variability was high in group 1 (table 2). Figure 2 shows that median PSA decreased with an increased dose.

Also for PV a statistically significant decrease compared to baseline was measured (table 2 and fig. 3). Considering data on all patients in LPC-002, the maximum PV reduction occurred at week 16 (mean 15.2\%). Six of the 20 responders showed more than a 20\% PV nadir. Also in LPC-003 PV decreased $13.1 \%$ at week 6 in group 1 but only $1.4 \%$ in group 2 at week 8 because 1 of 5 patients showed a great increase in PV.

The mean testosterone serum concentration before treatment in part 1 of LPC-002 and in group 1 in LPC-003 was 15.8 and $14.3 \mathrm{nmol} / \mathrm{l}$, respectively. In LPC-002 and LPC-003 the serum testosterone concentration at week 4 was increased $11.6 \%$ and $15.2 \%$, respectively. Thereafter the levels remained constant or returned to baseline.
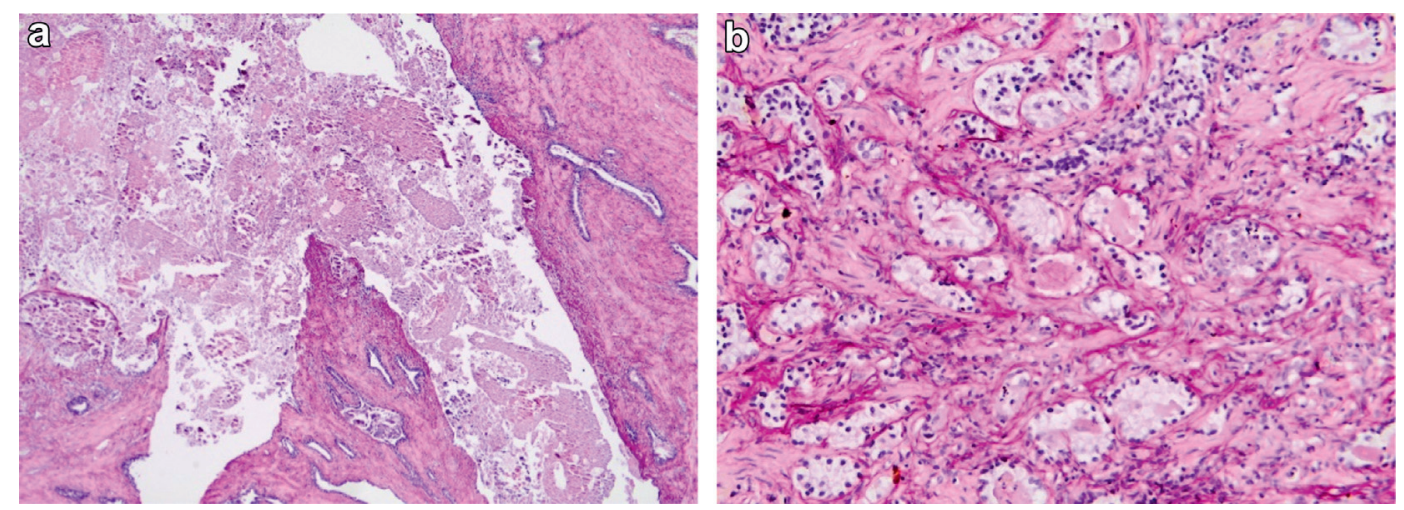

Figure 4. $a$, amorphous material with remaining drug in prostatic tissue. Note foreign body reaction. $b$, treated and affected cancerous prostatic tissue. Note shrunken nuclei and clear cytoplasm. Sirius positive, reduced from $\times 40$. 


\section{Histopathology Findings}

Table 3 summarizes LPC-003 histological results. There was a partial increase in the cytoplasm-tonuclear ratio at week 6 in 10 of 18 patients compared to before treatment and 10 patients also showed clear cytoplasm. Overall 12 of 18 patients showed histopathological changes, including a stroma reduction, as evaluated in prostate tissue after prostatectomy. In group 2 only 1 of 5 patients showed histopathological changes. Figure $4 \mathrm{dem}-$ onstrates typical histological effects, which resembled those of oral antiandrogen. ${ }^{18}$

\section{Magnetic Resonance Imaging, Magnetic Resonance Spectroscopy and 2-Dimensional Magnetic Resonance Spectroscopic Imaging}

Of the 23 patients who underwent MRI in LPC-003 19 were evaluable. The median size of detected measurable lesions in the prostate was $14 \mathrm{~mm}$ in the transverse plane on T2W images. A total of 12 and 4 patients in groups 1 and 2, respectively, had a measurable lesion at week 6 . None of the lesions demonstrated a measurable change in size only at 6 to 8 weeks.

Table 3 summarizes MRI changes. Decreased $\mathrm{PCa}$ volume in the peripheral zone and decreased overall signal intensity on $\mathrm{T} 2 \mathrm{~W}$ images were found in 6 of 15 patients in group 1 and in 2 of 4 in group 2 , indicating a global effect. A contrasting decrease between the lesion and surrounding peripheral zone tissues on T2W images was observed in 11 of 12 evaluable patients in group 1 and in 3 of 4 in group 2, indicating a tumor effect. A total of 14 patients per group showed overall changes on T2W images. The ADC of index lesions before treatment and at the week 6 followup did not significantly a

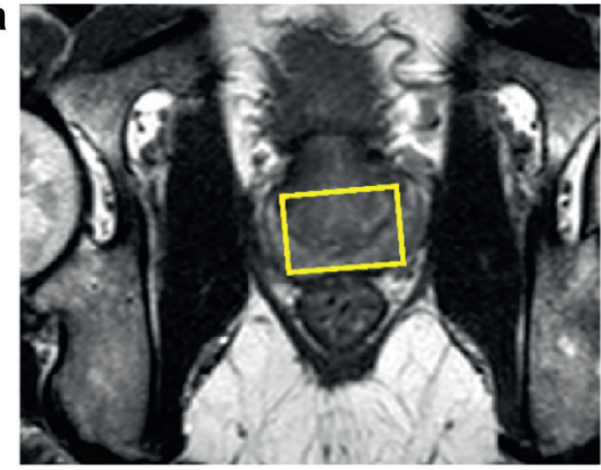

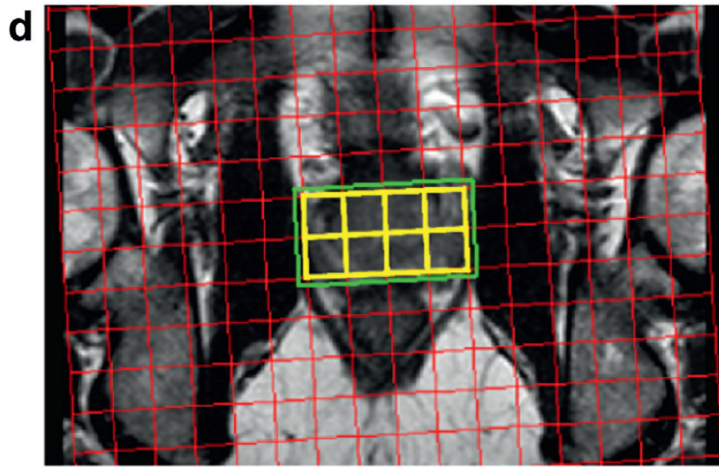

b

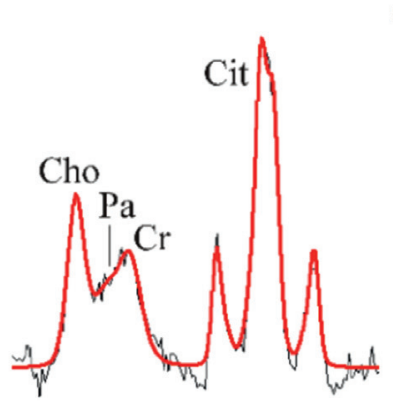

e

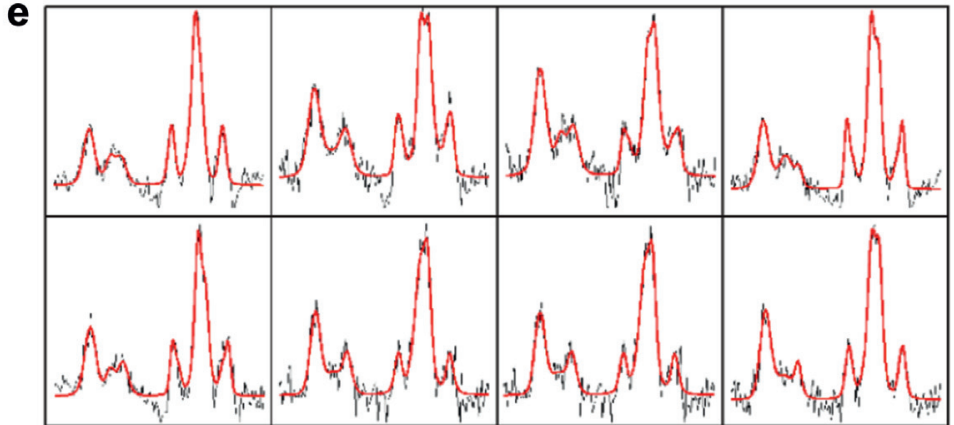

f

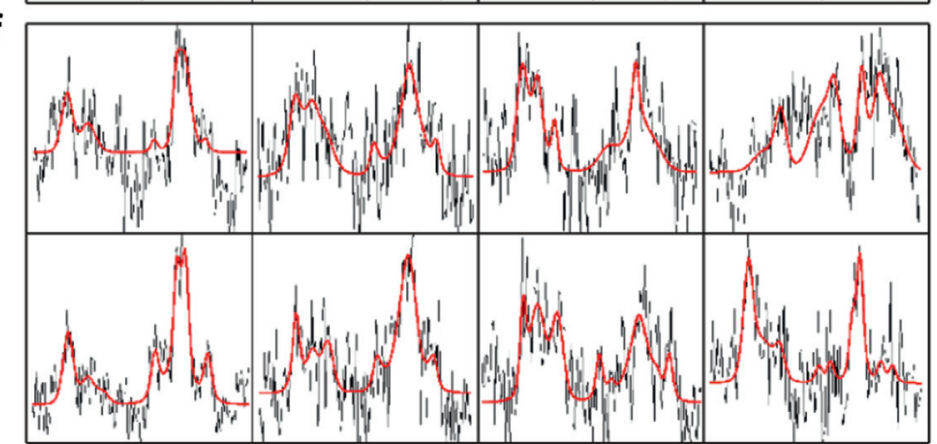

Figure 5. MR spectra in representative patient prostate. a to $c$, single voxel MRS. $d$ to $f, 2 \mathrm{D}$ MRSI. $a$, typical voxel position in axial plane (yellow rectangle). $b$, spectrum before treatment with Cit content $16.2 \mathrm{mM}$. $c$, spectrum after treatment with Cit content 6.7 mM. $d, 2 \mathrm{D}$ MRSI voxels (yellow squares) in axial plane. $e$, spectra before treatment. $f$, spectra after treatment. 
differ ( $p>0.05)$. It should be noted that ADC values could not be evaluated in 8 patients due to formulation matrix and/or blood at the site of the index lesion.

Figure 5 shows MR spectra in a representative patient before and after treatment. The spectral intensity of Cho, PA, $\mathrm{Cr}$ and Cit decreased. The effect was seen in benign as well as in PCa tissues. Consequently the SNR decreased. The Cit concentration decreased more than Cho and $\mathrm{Cr}$ levels, resulting in an increase in the $(\mathrm{Cho}+\mathrm{PA}+\mathrm{Cr}) / \mathrm{Cit}$ ratio. Single voxel MRS before and after treatment could be completed in 5 patients in LPC-003 group 1 . In these patients the median (Cho $+\mathrm{PA}+\mathrm{Cr}$ )/ Cit ratio increase was $71 \%$ (range $12 \%$ to $110 \%$ ) and the median SNR decrease was $44 \%$ (range $22 \%$ to $80 \%$ ).

\section{Safety}

In LPC-002 quality of life was unaffected and there were no major changes in voiding symptoms. Laboratory data were normal or clinically insignificant. There were 23 adverse events in LPC-002 and 24 in LPC-003, of which the most common were hematuria, urinary retention, hemospermia and urinary tract infection. Three serious adverse events were reported, including 2 prostate infections in LPC002 , and 1 sepsis and pelvic pain event caused by urinary retention in LP-003. All resolved with antibiotic treatment.

\section{Pharmacokinetics of 2-Hydroxyflutamide}

In LPC-002 part 1 the terminal half-life determined by release from the depot was 10.6 days. The maximum 2-HOF plasma concentration was $83 \mathrm{ng} /$ $\mathrm{ml}$ at a mean half-life of 2.2 days after injection but it remained measurable at 1 to $10 \mathrm{ng} / \mathrm{ml}$ for about 3 months after injection. Plasma exposure measured by the mean AUC was $1,280 \mathrm{ng} / \mathrm{ml}$ per day.

\section{DISCUSSION}

These 2 clinical studies revealed that the study formulation with 2-hydroxyflutamide in a NanoZolid carrier after intraprostatic injection was well tolerated in patients with localized $\mathrm{PCa}$ within the studied dose ranges. No hormone related side effects or other quality of life related effects were recorded. Adverse events were similar to those of transrectal prostate biopsy, ie urinary disorders and infections. ${ }^{19,20}$

Reductions in average PSA and PV were observed, indicating that androgen receptor mediated processes in the prostate gland were affected. The increased dose in LPC-003 resulted in a further decrease in PSA at week 6 or 8 while it is expected to increase further with treatment time in LPC-002. PSA and PV fluctuated considerably, similarly to previously described values. ${ }^{21}$

Several factors contribute to individual variations in effects on PSA and PV. The injection may intermittently cause leakage of intratissue PSA. $\mathrm{PV}$ is increased since the injected volume contributes to the gland. The formulation was administered only locally and near observed lesions. It was reported that 6 or 8 weeks is an insufficient drug exposure time to produce significant changes in histopathology, which are more pronounced after 3 months. ${ }^{22}$

Treatment effects were visible on T2W images as lower signal intensity in the whole prostate and as decreased PCa tissue contrast compared to surrounding normal tissues. Full pretreatment and posttreatment MRS data applicable for quantitative evaluations were available on only 5 patients in LPC-003 group 1 at Uppsala University Hospital. The increase in the $(\mathrm{Cho}+\mathrm{PA}+\mathrm{Cr}) /$ Cit spectral intensity ratio and the SNR decrease after treatment were noted in all 5 patients. These results suggest antitumor treatment effects. Also, the decrease in whole prostate volume indicates cell atrophy.

Plasma exposure of 2-HOF was substantially lower than that of oral flutamide of similar doses, which are in the order of a maximum concentration of $1,700 \mathrm{ng} / \mathrm{ml}$ and an AUC of 8,400 ng/ml per hour ${ }^{23}$ compared to 72 to $83 \mathrm{ng} / \mathrm{ml}$ and 1,280 to $1,365 \mathrm{ng} / \mathrm{ml}$ per hour in LPC-003.

Provided that an escalated dose results in a PSA reduction exceeding $50 \%$ to $60 \%$ without side effects, a possible future target group for this less invasive treatment is men on active surveillance who are at risk for disease progression. The therapy may treat coexistent, occult higher grade cancer, decrease the need for radical therapy to less than $40 \%$ in 10 years and reduce the risk of metastatic progression to less than $5 \%$ in 15 years, as currently associated with this group. ${ }^{5,9}$

\section{CONCLUSIONS}

Liproca Depot intraprostatic injections in patients with localized PCa showed average PSA and PV reductions and indications of antitumor effects. A dose-effect relationship was also observed. However, the data spread was significant and some patients were nonresponders. The antiandrogen related side effects, typical of those for oral use of the same drug, were absent. For the evaluated doses and study durations Liproca Depot was safe and tolerable. For better anticancer effects higher doses and a better dose distribution are suggested. 


\section{REFERENCES}

1. Fitzmaurice C, Allen C, Barber RM et al: Global, regional, and national cancer incidence, mortality, years of life lost, years lived with disability, and disability-adjusted life-years for 32 cancer groups, 1990 to 2015: a systematic analysis for the Global Burden of Disease Study. JAMA Oncol 2017; 3: 524.

2. Cancer Facts and Figures 2016. Atlanta: American Cancer Society 2016.

3. Sieh W, Lichtensztajn DY, Nelson DO et al: Treatment and mortality in men with localized prostate cancer: a population-based study in California. Open Prostate Cancer J 2013; 6: 1.

4. Schröder FH, Hugosson J, Roobol MJ et al: Screening and prostate-cancer mortality in a randomized European study. ERSPC Investigators. N Engl J Med 2009; 360: 1320.

5. Klotz L: Active surveillance for prostate cancer: debate over the application, not the concept. Eur Urol 2015; 67: 1006

6. Ahmed HU: The index lesion and the origin of prostate cancer. N Engl J Med 2009; 361: 17.

7. Murphy JC, Srinivas S, Terris MK et al: Flutamide administration at $500 \mathrm{mg}$ daily has similar effects on serum testosterone to $750 \mathrm{mg}$ daily. J Androl 2004; 25: 630.

8. Sanda MG, Dunn RL, Michalski J et al: Quality of life and satisfaction with outcome among prostate-cancer survivors. N Engl J Med 2008; 359: 200.
9. Klotz L, Vesprini D, Sethukavalan P et al: Longterm follow-up of a large active surveillance cohort of patients with prostate cancer. J Clin Oncol 2014; 33: 272.

10. Michaelson MD, Cotter SE, Gargollo PC et al: Management of complications of prostate cancer treatment. CA Cancer J Clin 2008; 58: 196.

11. Goya N, Gotanda K, Sasaki Tet al: Local injection of a sustained-release antiandrogen formulation into a target prostatic site: an experimental study. BJU Int 2007; 99: 202.

12. Agrawal SK, Agarwal MM and Singh SK: Intraprostatic injection of botulinum toxin: a promising treatment for patients with benign prostatic hyperplasia. Indian J Urol 2009; 25: 421.

13. Vezdrevanis K: Prostatic carcinoma shrunk after intraprostatic injection of botulinum toxin (case report). Urol J 2011; 8: 239.

14. Thomas MV and Puleo DA: Calcium sulfate: properties and clinical applications. J Biomed Mater Res B Appl Biomater 2009; 88: 597.

15. Sjogren E, Tammela TL, Lennernäs B et al: Pharmacokinetics of an injectable modified-release 2-hydroxyflutamide formulation in the human prostate gland using a semiphysiologically based biopharmaceutical model. Mol Pharm 2014; 11: 3097

16. Van Andel G, Bottomley A, Fosså SD et al: An international field of the EORTC QLQ-PR25: a questionnaire for assessing quality of life of patients with prostate cancer. Eur J Cancer 2008, 44: 2418.

17. Weis J, Jorulf $H$, Bergman A et al: MR spectroscopy of the human prostate using surface coil at $3 \mathrm{~T}$ : metabolite ratios, age-dependent effects, and diagnostic possibilities. J Magn Reson Imaging 2011; 34: 1277.

18. Civantos F, Manuel A and Marcial M: Pathology and androgen deprivation therapy in prostate carcinoma-a comparative study of 173 patients. Cancer 1995; 75: 1634.

19. Rosario DJ, Lane JA, Metcalfe $C$ et al: Short term outcome of prostate biopsy in men tested for cancer by prostate specific antigen: prospective evaluation with ProtecT study. Br Med J 2012; 344: d7894.

20. Lundström KJ, Drevin L, Carlsson $S$ et al: Nationwide population based study of infections after transrectal ultrasound guided prostate biopsy. J Urol 2014; 192: 1116.

21. Eastham JA, Riedel E, Scardino PT et al: Variation of serum prostate-specific antigen levels: an evaluation of year-to-year fluctuations. JAMA 2003; 289: 2695.

22. Montironi R and Schulman C: Pathological changes in prostate lesions after androgen manipulation. J Clin Pathol 1998; 51: 5.

23. Schulz M, Schmoldt A and Donn F: The pharmacokinetics of flutamide and its major metabolites after a single oral dose and during chronic treatment. Eur J Clin Pharmacol 1988; 34: 633. 